\title{
Comparison of aspiration VS non- aspiration techniques in fine- needle cytology of thyroid lesions
}

\author{
Dr. SwaranKaur ${ }^{1}$, Dr Uma Garg ${ }^{2}$,Dr Satwant Kaur ${ }^{3}$, Dr Kulwant Singh ${ }^{4}$ \\ DRHemlata Kamra ${ }^{5}$, DR Sanjay Verma ${ }^{6}$ \\ ${ }^{1}$ Prof \&HOD Pathology BPS GMC for women, KhanpurKalan-Sonepat ${ }^{2}$ Prof \&HOD ENT BPS GMC for \\ women, KhanpurKalan-Sonepat, ${ }^{3}$ Assoc.Prof. OBG MMMC \& H Kumat Hatti Solan \\ ${ }^{4}$ Assoc. Prof.Pathology BPS GMC for women, KhanpurKalan-Sonepat $5 \& 6$ Asst. Proff,.Pathology BPS GMC \\ for women, KhanpurKalan-Sonepat
}

\begin{abstract}
AIM: To compare the efficacy of fine - needle non - aspiration cytology (FNNAC) with that of fine - needle aspiration cytology (FNAC) of thyroid lesions.

Materials and Methods: FNAC and FNNAC techniques were studied in 88 cases of thyroid lesions. All the needle - sampling procedures were done by a single operator. The samples were assessed cytologically and evaluated using five parameters, that is, background blood or clot, amount of cellular material, degree of cellular degeneration, and degree of cellular trauma and retention of appropriate architecture.

Statistical Analysis: Wilcoxon signed rank test was performed using SPSS 14 software. Differences between all the individual parameters as observed in FNAC and FNNAC smears were insignificant.

Results and Conclusion: After evaluation of FNAC and FNNAC on the basis of these scores, greater numbers of diagnostically superior samples were obtained by FNNAC; however, by FNAC more number of diagnostically adequate smears were observed. The numbers of unsuitable smears were also more by FNNAC technique.
\end{abstract}

Key words: Thyroid lesion; FNAC; FNNAC.

\section{Introduction}

Fine - needle aspiration cytology (FNAC) for tumors was first described by Martin and Ellis in the year 1930 in the United States. In vascular organs, an alternative method of fine - needle non - aspiration cytology (FNNAC) also known as cytopuncturewas developed in France in 1982 by Brifford et al ${ }^{(1)}$. This study was undertaken to compare the efficacy and quality of FNNAC with that of FNAC of thyroid les ion using both techniques in 88 thyroid lesion to ascertain whether it could be chosen as a superior cytodiagnostic procedure in vascular organs, especially the thyroid.

\section{Materials and Methods}

The study population comprised all patients who presented with thyroid swelling at the Department of pathology (cytology section) from Dec 2009 to Nov 2011 at Muzaffarnagar Medical College, Muzaffarnager . After a thorough clinical examination, all the patients were subjected to both FNAC and FNNAC. A total of 50 cases of thyroid lesions were included in this study. The procedure was explained to the patient and verbal consent was obtained prior to performing the procedure. The patients were subjected to FNAC and FNNAC using 23 - gauge needles and 10 - cc plastic syringes. All the procedures were performed by a single operator. FNAC or FNNAC sampling was carried out randomly with lesions, irrespective of consistency and size of lesion. Every slide was assessed without the prior knowledge of techniques utilized. The study was thus single blind and also prevented the observer bias. The smears were scored according to criteria using a predetermined scoring developed by mair etal ${ }^{(2)}$. The two sampling techniques were compared using five objective parameters : (1) background blood or clot; (2) amount of cellular material; (3)degree of cellular degeneration; (4) degree of cellular trauma; and (5) retention of appropriate architecture.(Table 1)

A cumulative score between 0 and 10 points was obtained for each specimen which was then categorized into one of the following three categories :

Category 1 - (Score 0-2) Unsuitable for diagnosis.

Category 2 - (Score 3-6) Adequate for cytological diagnosis.

Category 3 - (Score 7-10) Diagnosis superior. 


\section{Statistical analysis}

The difference in the score for the individual parameter was assessed by Wilcoxon signed rank test using SPSS14 software. All the results were analyzed considering the statistical significance at a level of $\mathrm{P}=$ 0.05 .

\section{Results}

The non - aspiration technique yielded less diagnostically adequate but more diagnostically superior smears when compared with aspiration technique. A total of 19 cases were unsuitable for cytodiagnosis by non - aspiration as compared with 17 cases by aspiration technique. (Table 2)

$\mathrm{P}$ value obtained by Wilcoxon singed rank test was not statistically significant in favour of no - aspiration sampling for any parameter. However, the average scores for each parameter favoured non - aspiration sampling than aspiration sampling. (Table 3)

Non - aspiration sampling displayed more cellular material, less

Table 1 : Table of point allocation ${ }^{(2)}$

Criteria Quantitative description Point score

Background blood/

Clot Large amount; great compromise of diagnosis

Moderate amount; diagnosis possible

Minimal; diagnosis

Amount of cellular

Minima to absent; diagnosis not possible

Material

Sufficient for cytodiagnosis

Abundant; diagnosis possible

Degree of cellular Marked; diagnosis impossible

possible

12

Minima; diagnosis easy

Degree of cellular

Marked; diagnosis impossible;

Trauma

Moderate; diagnosis possible

Minimal; diagnosis obvious

$\begin{array}{crr}0 & 1 & \\ & 2 & \\ \text { Degeneration } & \text { Moderate; diagnosis }\end{array}$

0
Retention of Appropriate Architecture
Minimal to absent non - diagnostic

Moderate; some preservation of, e.g., follicle, papillae, acini, etc.

Excellent architectural display closely reflecting

Histology; diagnosis obvious

Table 2 : The performance of FNAC and FNNAC technique

Performance

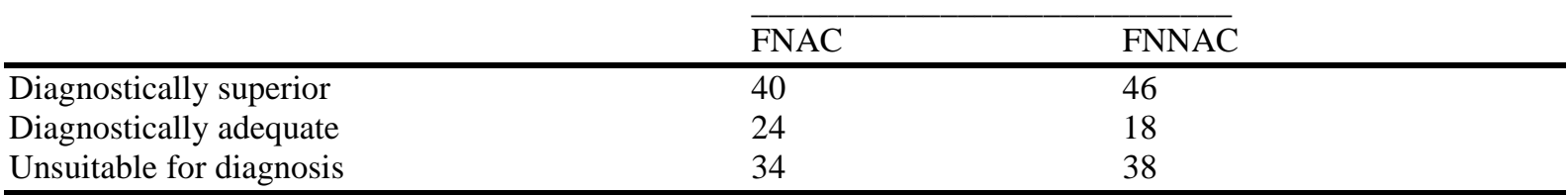
parentheses are in percentage

Cellular trauma and degenerative changes, better retention of architecture and less likelihood of obscuring by blood. The average score per case was 6.04 by non - aspiration technique and was 5.90 by aspiration technique. Table 4 depicts the frequencies of the various lesions encountered.

Discussion

FNAC, since its inception in 1847 , has passed through two phases of initial scepticism and interim enthusiasm and has successfully reached the final stage of acceptance as identified by Orell ${ }^{(3)}$ in his analysis of steps by which the assessment of innovative diagnostic practice progresses. FNAC is widely accepted as the primary method for diagnosis of thyroid lesions. The cytologist faced the common problem in interpreting the 
hemorrhagic material from thyroid obtained by $\mathrm{FNAC}^{(4)}$. To overcome this inherent problem, an alternative technique FNNAC also called cytopuncture or fine - needle capillary sampling was used first in France for breast tumor and later for orbital and periorbital tumors. The thyroid gland, which is very vascular, often yields aspirate markedly admixed with blood. It has been suggested that FNNAC sampling, by eliminating the negative suction pressure employed in FNAC, decreases the dilution of thyroid cells by blood and the scientific basis was explained by Santos and Leiman ${ }^{(4)}$. Methods such as suction of material with a needle bore rely on the property of capillary tension in the narrow channel. The fluid or semi - fluid ascends into the narrow tube in inverse proportion to the diameter of that tube or capillary ${ }^{(5)}$.

Table 3 : Average score and $P$ value for each parameter

\begin{tabular}{lccc}
\hline Parameter & Aspiration sampling & Non - A P Value & \\
\hline & & & \\
Background blood or clot $>0.05$ & 1.16 & 1.24 & \\
Amount of cellular material $>0.05$ & 1.35 & 1.42 & 1.27 \\
Degree of cellular degeneration $>0.05$ & 1.18 & 1.32 & 1.13 \\
Degree of cellular trauma & $>0.05$ & 1.29 & 1.28 \\
Retention of appropriate architecture $>0.05$ &
\end{tabular}

Table 4 : Frequency of various thyroid lesions

\begin{tabular}{llc}
\hline Type of lesion & No. of cases & Percentage \\
\hline Multi nodular goitre & 24 & 27.27 \\
Thyroiditis & 18 & 20.45 \\
Colloid goitre & 16 & 18.18 \\
Colloid cyst & 10 & 11.33 \\
Follicular lesion & 10 & 11.33 \\
Follicular neoplasm & 6 & 6.81 \\
Toxic goitre & 2 & 2.27 \\
Papillary carcinoma & 2 & 2.27 \\
\hline Total & 88 & 100 \\
\hline
\end{tabular}

The important advantage of FNNAC sampling is easy operation and absolute control over operating hand, especially for neck, breast, cutaneous or subcutaneous tissue. The FNNAC also allows a better perception of tumor consistency.

Results when compared for background blood contamination supported the non - aspiration technique(Figures 1 and 2) but were not statistically significant. Similar to the study by Meherbano et $\mathrm{al}^{(6)}$.., the amount of cellular yield was found to be better by non - aspiration(Figures 3 and 4) but the difference was not statistically significant similar to study by Mair et al ${ }^{(2)}$. However, Jayaram and $\mathrm{Gupta}^{(7)}$ observed that cellularity was higher in aspiration smears than in non - aspiration smears in most of the goitres. Cellular degeneration ( Table 3 ) was greater in aspiration similar to the study done by Ghosh et $\mathrm{al}^{(8)}$, but the difference was not statistically significant. The degree of cellular trauma was less in non - aspiration as compared with aspiration (Figures 3 and 4) similar to the study done by Ghosh et al ${ }^{(8)}$. But the difference was not statistically significant.Non - aspiration smears yielded better retention of architecture with similar findings reported by others. For the five parameters studied objectively, there was no statistically significant difference observed between the two techniques, and a similar result has been found by Haddadi - Nezhad et al ${ }^{(9)}$. Whereas other observed a statistically significant difference in the total score in favour of non - aspiration as compared with aspiration technique, FNNAC producing a better quality of specimen. ${ }^{(10,11)}$

In the present study, more diagnostically superior and less diagnostically adequate sample were obtained more by non - aspiration technique in comparison to aspiration technique. It was observed that the percentage of inadequate sampling was more with non - aspiration (38\%) than with aspiration (34\%) technique in contrast to the observations of Santos and Leiman ${ }^{(4)}$ and Ciatto et $\mathrm{al}^{(12)}$. With the utilization of both the techniques for each case, the inadequacy was reduced to $12 \%$ as compared with $9.5 \%$ observed by Kamal et al. $^{(6)}$ 


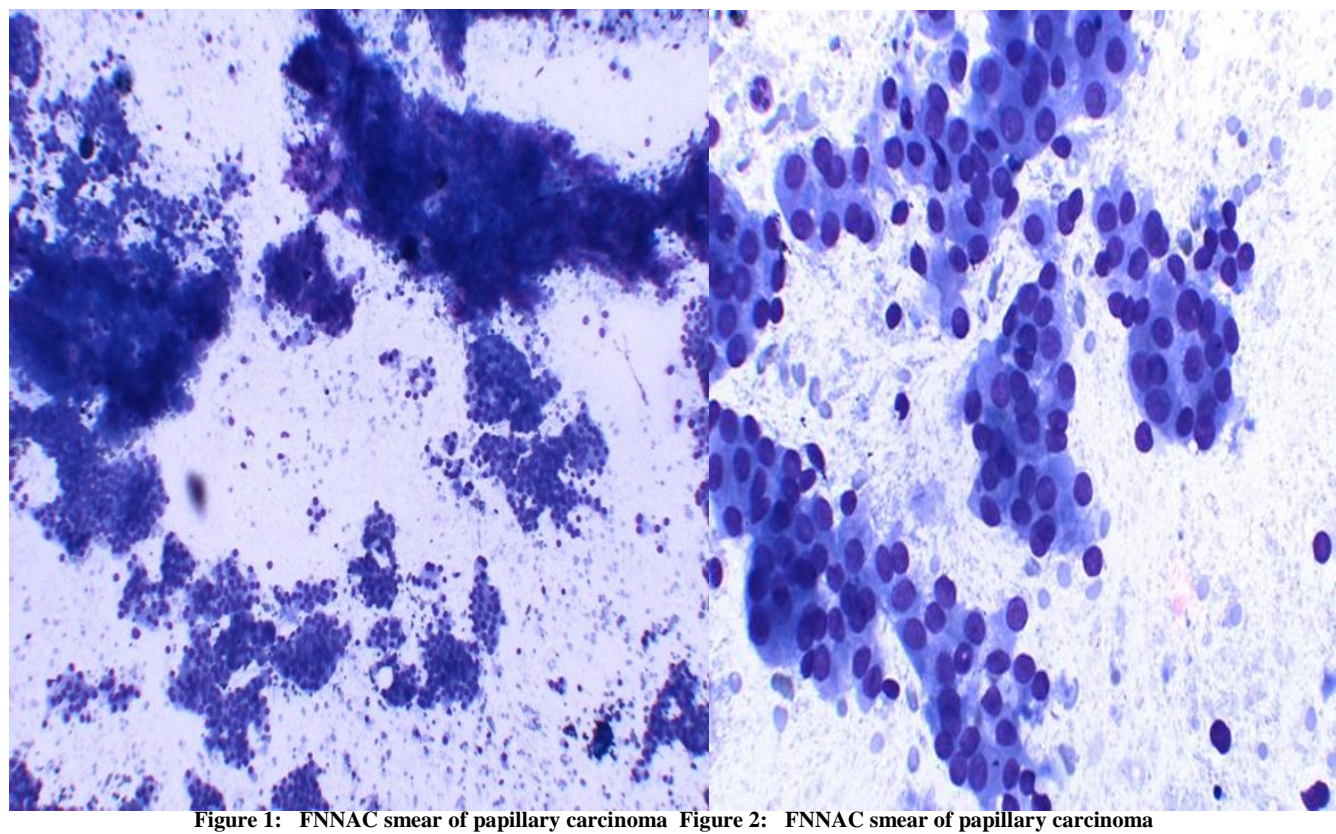

Thyroid (Leishman-Giemsa.X200)Thyroid (Leishman-Giemsa, x400)

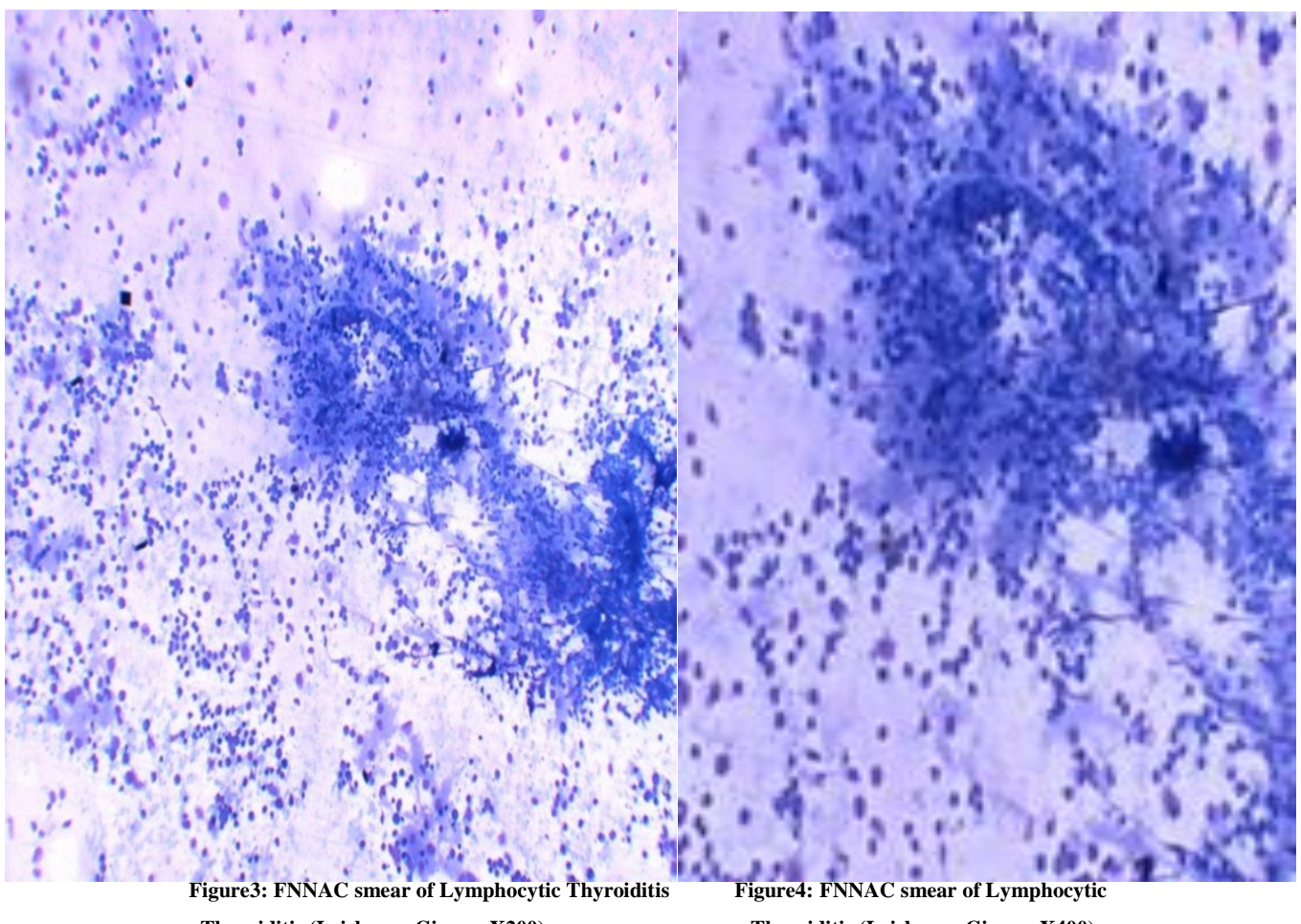

Thyroiditis (Leishman-Giemsa.X200)

Thyroiditis (Leishman-Giemsa.X400)

\section{Conclusions}

Both the techniques have their own merits and demerits the following three categories :By combining both the techniques, better diagnostic accuracy can be achieved. However, FNNAC technique is easier to perform with better patient compliance.

\section{References}

[1]. Zajdela A, Zillhardt, Voillemet N. Cytological diagnosis by fine needle sampling without aspiration. Cancer 1987; $59 ; 1201-5$.

[2]. Mair S, Dunbar F, Becker PJ, Du Plessis W. Fine needle cytology : is aspiration suction ? A study of 100 masses in various sites. ActaCytol 1989; 33: 809-13.

[3]. Orell SR. Fine needle aspiration in perspective. Pathology 1982; 14: 113- 44.

[4]. Santos JEC, Leiman G. Non aspiration fine needle cytology : Application of a new technique to nodular thyroid diseases. ActaCytol; 1988:32:353 - 56. 
[5]. Papanicolaou GN, Traut HF. Diagnosis of uterine cancer by the vagina smear. The commonwealth Fund, New York, 1943.

[6]. Meherbano MK, Arjune DG, Kulkarni HR. Comparative study of fine needle aspiration and fine capillary sampling of thyroid lesions. ActaCytol; 2002,46:30 - 34 .

[7]. Jayaram G, Gupta B. Non aspiration fine needle cytology in diffuse and nodular thyroid lesion : A study of 220 cases. ActaCytol ; 1991. 155:1217- 19.

[8]. Ghosh A, Mishra RK, Sharma SP, Singh HN, Chaturvedi AK. Aspiration vs non - aspiration technique of cytodiagnosis - A Critical evaluation in 160 cases. Indian J PatholMicrobiol; 2000. $43: 107-12$.

[9]. Haddadi - Nezhad S, Larijani B, Tavangar SM, Nouraei SM. Comparative of fine non - needle aspiration with fine needle aspiration technique in cytological studies of thyroid nodule. EndocrPathol; 2003. 14:369-73.

[10]. Dey P, Shashirekha, Ray R. Fine needle sampling without suction in intra - abdominal lesion. ActaCyta 1994;38:495-97.

[11]. Romitelli F, Di Stasio E, Santoro C, lozzino M, Orsini A, Cesareo R. A comparative study of fine needle aspiration and fine needle non - aspiration biopsy on suspected thyroid nodules. EndocrPathol 2009;20:108-13.

[12]. Ciatto S, Lossa A, Cicchi P et al : Non aspiration fine needle cytology of thyroid tumors. ActaCytol 1989;33:939. 